\title{
Genetic diversity in tetraploid populations of the endangered daisy Rutidosis leptorrhynchoides and implications for its conservation
}

\author{
ANTHONY H. D. BROWN* \& ANDREW G. YOUNG \\ Centre for Plant Biodiversity Research, CSIRO Plant Industry, GPO Box 1600, Canberra, ACT 2601, Australia
}

\begin{abstract}
Polyploidy is an important variable in assessing the genetics of endangered plant species. Species consisting of populations with different chromosome numbers pose questions as to the mode of inheritance, relative variability status, population divergence and gene flow. The self-incompatible species Rutidosis leptorrhynchoides (Asteraceae) in south-eastern Australia is a good example. The remnant populations in the northern sector of the species range are diploid, whereas southern ones are either diploid or tetraploid. Allozyme analysis of the tetraploid populations showed tetrasomic inheritance confirming an autopolyploid genetic system, a modest increase in their allelic richness over diploid populations in the same region and a lack of genetic divergence. Conservation and replenishment strategies should take account of these genetic features of mixed ploidy.
\end{abstract}

Keywords: allelic richness, allozyme polymorphism, autotetraploidy, conservation genetics, glucosephosphate isomerase, tetrasomic inheritance.

\section{Introduction}

Polyploidy is widespread in the plant kingdom - over half the species of higher plants are recent or ancient polyploids (Leitch \& Bennett, 1997). Furthermore, the frequency of polyploids is even higher among species such as weeds and crops, species that are currently thriving in environments modified by humans. This is a testament to the resilience of polyploid genomes. Several recent studies of polyploid plants have emphasized that the formation of polyploids is a continually repeating process, at rates akin to those of genic mutation (Ramsey \& Schemske, 1998).

Despite its prevalence and its effects on fitness, polyploidy is a relatively neglected feature of plant species when their conservation genetics are under discussion. The existence of polyploid populations, and particularly of populations with a mixed ploidy status, in plant taxa of conservation interest raises many genetic issues for attention. What should be the conservation status of polyploid populations? Ranker \& Arft (1994) considered whether allopolyploid species qualify as species under the United States Endangered Species Act because of their hybrid origin. They argue that

*Correspondence. E-mail: t.brown@pi.csiro.au protection should be extended to all 'self-reproducing hybrid species'. But how many such species deserve special protection? If, for example, they are allopolyploids that have arisen recently or recurrently from existing secure diploid species, the need to protect each distinct lineage may well be questioned. Similarly, if the criterion of self-reproducibility is invoked for autopolyploid populations, does each separate lineage merit individual conservation? More cases of polyploid taxa with multiple origins are coming to light (Soltis \& Soltis, 1999) and this implies that in the longer term new polyploid taxa can arise to replace lost older ones.

From a genetic standpoint, the first question to resolve concerns the nature of segregation in the polyploid. Whether the inheritance is predominantly disomic or tetrasomic has a marked effect on the generation of multilocus genotypic diversity.

A second set of questions concerns how the variability status of polyploid populations should be assessed. What is the level of diversity in polyploid populations? Several studies have found that heterozygosity and allelic richness (the number of alleles per locus) are higher in autoploids compared with their closely related diploids (Hokanson \& Hancock, 1998), and high in some allopolyploid species (e.g. Microseris lanceolata, Prober et al., 1998). Other studies, however, report no change in 
allelic richness with autopolyploidy (e.g. in Antennaria species by Bayer, 1989). Several allotetraploid species have low levels of polymorphism (e.g. Glover \& Abbott, 1995; Conte et al., 1998). Arft \& Ranker (1998) stated that the allotetraploid orchid Spiranthes diluvialis had a high percentage of polymorphic allozyme loci $(>50 \%$ loci), equal to related diploids in allelic richness. However, this estimate included a large amount of so-called 'fixed heterozygosity', or fixed differences between homoeologous loci in the contributing diploid genomes. A further complication to comparative studies concerns the number of gene copies in polyploid populations and in experimental samples, which is clearly higher than in diploid samples of the same size. Does this sampling effect bias the comparison of allelic richness in polyploids with that in related diploids? What is the variability status of polyploid populations over and above any increase arising from this sampling effect?

Thirdly, if both polyploid and diploid populations of a taxon are to be conserved, should geographical isolation between ploidy levels be enforced to avoid dysgenesis? One option is to ignore the cytological diversity; another option is to foster divergence, and eradicate and quarantine polyploids away from diploid populations. On the other hand, polyploidy, with its buffering effect on allelic richness and heterozygosity may be a mechanism to improve the chance of survival of a species in decline. Does polyploidy represent a method for increasing the conservation potential of a species? If so, is this a reason to bias the conservation strategy in favour of polyploid populations?

The cytologically complex, endangered daisy Rutidosis leptorrhynchoides F. Muell. (Asteraceae), the Button Wrinklewort, is an ideal species in which to explore these issues. The species is an under-storey component of the severely depleted grassy woodland ecosystem in southeastern Australia, and remnant populations of this daisy have been managed without information on its cytological complexity (Cropper, 1993). Chromosome numbers in the species are: $2 n=22,44$ and rarely $2 n=33,26$ and 52 together with various aneuploids 21-46. Cytogenetics and gene flow studies in the species are reported elsewhere (Young \& Murray, 2000). Our specific purposes here are to confirm tetrasomic inheritance as suggested by the cytological evidence; and to estimate levels of genetic diversity and population divergence in tetraploid populations $(2 n=4 \mathrm{x}=44)$ of $R$. leptorrhynchoides compared with their diploid counterparts.

\section{Materials and methods}

Rutidosis leptorrhynchoides is a multistemmed, selfincompatible, herbaceous perennial daisy endemic to the grassland and grassy woodland communities of south-eastern Australia. This is a highly fragmented ecosystem with only about $0.5 \%$ of the original two million hectares of this ecosystem remaining after 150 years of rural development. Herbarium records point to a marked reduction in the number, size and geographical spread of populations of this species. The species is listed as nationally endangered (Briggs \& Leigh, 1995) and is confined to some 24 populations in remnant vegetation. On a geographical scale, the remnant populations are in two clusters. The northern cluster $\left(<35^{\circ} 30^{\prime} \mathrm{S}\right.$, $>148^{\circ} 30^{\prime} \mathrm{E}$ ), in New South Wales and the Australian Capital Territory, consists almost entirely of diploid individuals $(2 n=22)$. The southern cluster $\left(>37^{\circ} \mathrm{S}\right.$, $<145^{\circ} 30^{\prime} \mathrm{E}$; central Victoria) includes both diploid and tetraploid $(2 n=44)$ populations. Tetraploid plants closely resemble the diploid plants in morphology both in the field and glasshouse. However tetraploids tend to have $20 \%$ fewer florets per capitulum, and pollen grains with approximately $30 \%$ larger diameter (Young et al., 2000).

\section{Populations, samples and starch gel electrophoresis}

The sampling, preparation of the material for assay, gel techniques and enzyme assays are those described and used in comparable studies of diploid populations of this species (Young et al., 1999). The five known Victorian populations that consisted largely or entirely of tetraploid individuals were sampled. Table 1 lists these populations, their co-ordinates, altitude and current population size, in comparison with the three Victorian diploid populations. These diploid populations form a group on the Werribee coastal plain about $40 \mathrm{~km}$ west of Melbourne, whereas the tetraploids occur in more elevated sites on the southern slopes of the Western Highlands, south and west of Ballarat.

Five seeds from 35 maternal plants per population (except for the small population at Wickcliffe) were vernalized $\left(3\right.$ days at $\left.4^{\circ} \mathrm{C}\right)$ and germinated on moist paper for two weeks. Crude extracts from a single seedling from each of the 35 mothers (with additional seedlings from a few mothers for two sites) were subject to starch gel electrophoresis using three buffer systems, assaying nine loci with five enzyme assays:

1 Histidine-citrate system: glucose-6-phosphate isomerase, GPI, EC 5.3.1.9. (one locus), phosphoglucomutase, PGM, EC 5.4.2.2 (three loci), menadione reductase, MNR, EC 1.6.99 (one locus).

2 Lithium-citrate-borate system: aspartate aminotransferase, AAT, EC 2.6.1.1 (three loci).

3 Tris-citrate-borate system: alcohol dehydrogenase, ADH, EC 1.1.1.1 (one locus).

The enzymes AAT, ADH and GPI had a dimeric tertiary structure, whereas MNR was tetrameric and 
Table 1 Population size, geographical location and altitude for the five tetraploid and three diploid populations of Rutidosis leptorrhynchoides, in Victoria, Australia

\begin{tabular}{|c|c|c|c|c|}
\hline Population & $\begin{array}{l}\text { Reproductive } \\
\text { population size }\end{array}$ & $\begin{array}{l}\text { Geographical } \\
\text { coordinates }\end{array}$ & $\begin{array}{l}\text { Altitude } \\
\text { (metres) }\end{array}$ & $\begin{array}{l}\text { Other ploidy } \\
\text { level present? } \dagger\end{array}$ \\
\hline \multicolumn{5}{|l|}{ Tetraploids } \\
\hline Bannockburn & 340 & $38^{\circ} 04^{\prime} \mathrm{S} 144^{\circ} 11^{\prime} \mathrm{E}$ & 100 & No \\
\hline Dobies Bridge & 2616 & $37^{\circ} 19^{\prime} \mathrm{S} 143^{\circ} 01^{\prime} \mathrm{E}$ & 300 & No \\
\hline Middle Creek & 610 & $37^{\circ} 25^{\prime} \mathrm{S} 143^{\circ} 13^{\prime} \mathrm{E}$ & 360 & No \\
\hline Rokewood & 5419 & $37^{\circ} 54^{\prime} \mathrm{S} 143^{\circ} 43^{\prime} \mathrm{E}$ & 190 & $\begin{array}{l}\text { Yes } \\
\text { (one } 2 n=26 \\
\text { three } 2 n=39 \text { ) }\end{array}$ \\
\hline Wickcliffe & 28 & $37^{\circ} 41^{\prime} \mathrm{S} 142^{\circ} 43^{\prime} \mathrm{E}$ & 220 & No \\
\hline Geometric mean & 607 & & & \\
\hline \multicolumn{5}{|l|}{ Southern diploids } \\
\hline Manor & 13 & $37^{\circ} 56^{\prime} \mathrm{S} 144^{\circ} 35^{\prime} \mathrm{E}$ & 40 & $?$ \\
\hline St Albans & 137 & $37^{\circ} 45^{\prime} \mathrm{S} 144^{\circ} 48^{\prime} \mathrm{E}$ & 60 & No \\
\hline Truganina & 626 & $37^{\circ} 50^{\prime} \mathrm{S} 144^{\circ} 43^{\prime} \mathrm{E}$ & 40 & No \\
\hline Geometric mean & 104 & & & \\
\hline
\end{tabular}

$\dagger$ Unpublished data from Murray and Young.

PGM monomeric. Genotype scores were inferred in comparison with diploid samples and in all except the three $P g m$ loci, the gene dosage in diallelic or triallelic heterozygotes was scorable from the relative intensity of homomultimers and predicted heteromultimers. In the case of the Pgm loci, the allozyme array was scorable but not the gene dosage for all individuals. Therefore the $P g m$ loci were included in the estimate of polymorphism $(P)$ and allelic richness $(A)$, but estimates of heterozygosity, inbreeding coefficients and population divergence were based on only the six multimeric loci in both tetraploid and diploid populations.

Progeny for the inheritance studies were generated from specific crosses. Leaf tissue from plants established in the glasshouse and of known chromosome number was assayed for loci coding for multimeric isozymes (Gpi-2, Mnr-1, and Aat-1, Aat-2, Aat-3). Crosses that would yield segregation information were made by controlled pollination (Young \& Brown, 1999). The isozyme markers chosen for the ploidy inheritance test were those where every genotype could be recognized unambiguously, to minimize the chance of misclassification. More than 100 progeny from the most informative available cross were assayed as seedlings and scored. The observed numbers of progeny were tested against numbers expected under both disomic and tetrasomic models of inheritance.

\section{Data analysis}

Four measures of genetic diversity were estimated: the polymorphism $(P)$, or percentage of loci exhibiting more than one allele; the allelic richness $(A)$, or mean number of alleles present per locus; the observed heterozygosity $\left(H_{\mathrm{o}}\right)$; and the heterozygosity expected with random union of gametes $\left(H_{\mathrm{e}}\right)$. Observed heterozygosity of individual genotypes was scored following Bever \& Felber (1992), with values being one minus the probability that any two alleles drawn at random were identical by descent $(A A A A=0, A A A B=0.50$, $A A B B=0.66, A A B C=0.83$ and $A B C D=1)$. Expected heterozygosity was computed separately under the assumptions of either random chromatid segregation or random chromosome segregation (Allard, 1960). All estimates were calculated using the computer program AUTOTET (Thrall \& Young, 2000). Deviation from random mating was assessed using Wright's fixation index $(F)$. To examine patterns of genetic differentiation among populations Nei's genetic distance was calculated among all possible pairs of populations and used to perform a hierarchical cluster analysis (unweighted pairgroup method with arithmetic averaging - UPGMA) using Ritland's (1989) GD computer package.

\section{Results}

\section{Inheritance}

Table 2 summarizes the segregation observed for the isozyme locus $G p i-2$ in a controlled cross $(F G G M \times$ $M M M M)$ and its reciprocal $(M M M M \times F G G M)$. As these data are homogeneous they were added together. Adjacent columns list the number of genotypes expected under four models of inheritance. These are (1) disomic 


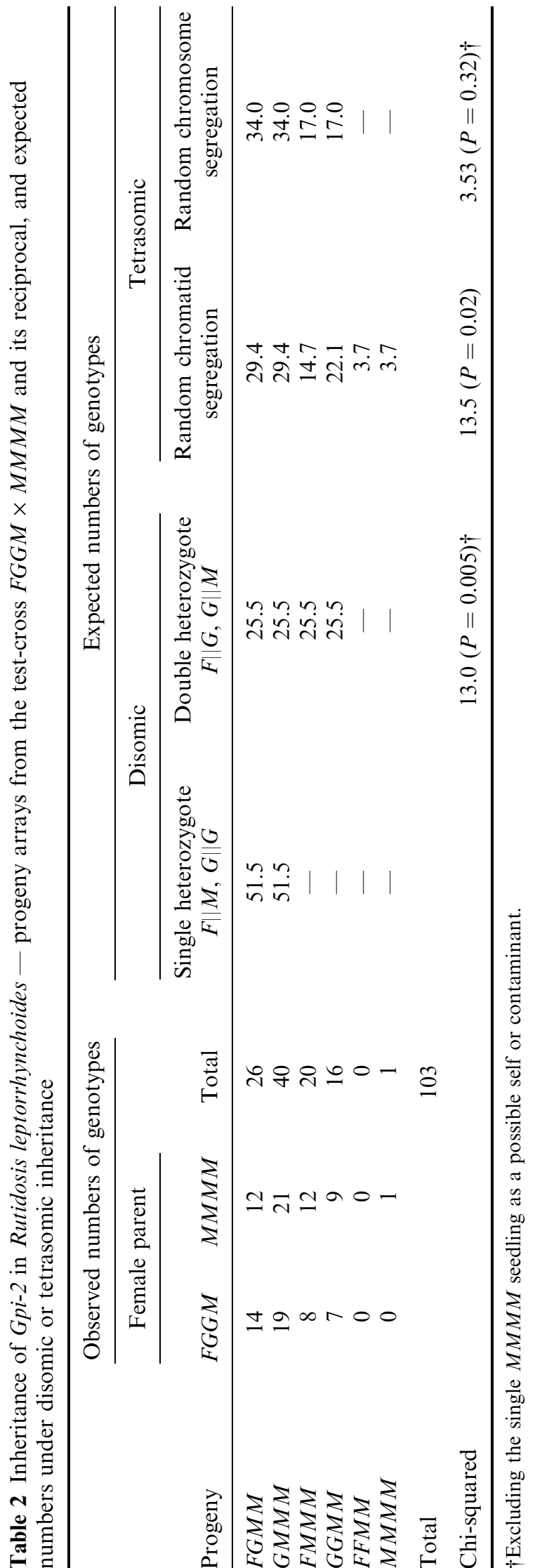

assuming the test genotype was heterozygous at one locus ( $F$ segregating from $M)$; (2) disomic assuming the test genotype is a double heterozygote $(F / / G$ and $M / / G)$; (3) tetrasomic with the maximum amount of double reduction as pertains when the marker locus recombines freely with the centromere, i.e. chromatid segregation; and (4) tetrasomic with no double reduction and the locus closely linked to the centromere, i.e. chromosome segregation.

As expected, the numbers of genotypes were similar in both directions of the cross. The recovery of one $M M M M$ individual progeny in this cross is diagnostic for the occurrence of tetrasomic inheritance with double reduction and hence decisive evidence of autopolyploidy. However, because this sole progeny was obtained when the female parent was of the same genotype (MMMM), there is the possibility that this seedling might be a rare instance of self-fertilization. Very rarely self-fertilized seed can be recovered in this otherwise highly selfincompatible species (Young \& Brown, 1999).

Leaving this individual aside, it is possible to test the segregation for the remaining 102 progeny for consistency with various segregation models. Because genotypes other than $F G M M$ and $G M M M$ were observed, the disomic-single heterozygote model is inapplicable. The chi-squared value for departure of the observed array from the disomic, double heterozygous segregation is $13.0(P=0.005)$, supporting rejection of this model. Thus both models of disomic segregation, that are typical for allopolyploids, fail to fit the data. In contrast, the observed ratio of the four classes of progeny agrees (chi-squared $=3.5, \quad P=0.3$ ) with that expected for tetrasomic inheritance for an autotetraploid with no double reduction. We infer that this marker locus is close to the centromere of the chromosome on which it is located. The locus does not segregate independently of the centromere because the data fail to fit the tetrasomic model with random chromatid segregation.

\section{Tetraploid population genetic structure}

Table 3 gives the estimates of genetic diversity for the five tetraploid populations in Victoria. Included for comparison are parallel estimates from the diploid populations from Victoria, and the average estimates from all the diploid populations covering both north and south regions. The diploid data are recomputed from Young et al. (1999) to include only those allozyme loci that are common to both the diploid and tetraploid studies (nine loci for estimates of $P$ and $A$; six loci for estimates of $H$ and $F$ ).

The allelic richness of the average tetraploid population (the observed number of alleles per locus) was 3.2, which was $30 \%$ and significantly higher than the allelic 


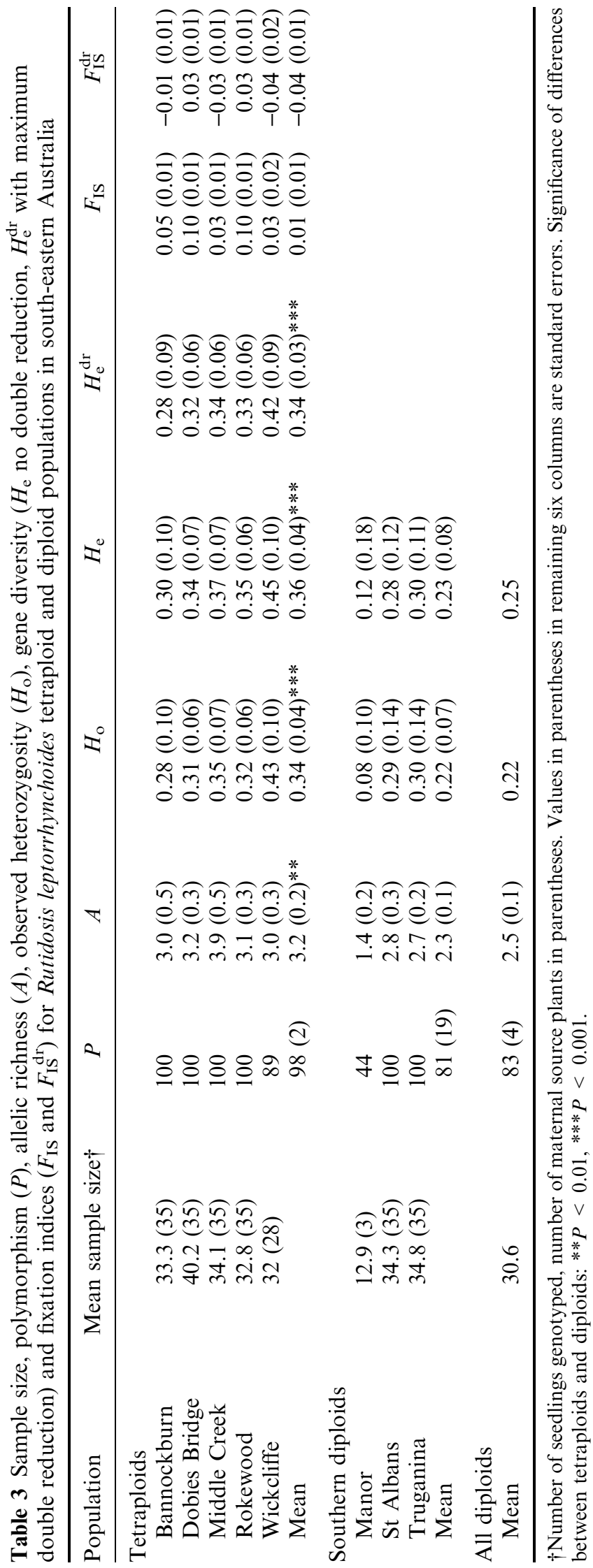

richness of the diploid populations. Figure 1 is a plot of allelic richness against the population size on a log scale for each population. The figure shows that richness of tetraploid populations exceeded that in diploids of comparable size. However, richness was not significantly related to the current size of these tetraploid populations, unlike the situation in diploids (Young et al., 1999).

The observed heterozygosity, as defined above, was substantially higher than that observed in diploid populations, indicating a greater capacity in tetraploids to generate genetic diversity among progeny. The expected heterozygosity computed assuming tetrasomic inheritance either with or without maximum double reduction was virtually identical to the observed, giving fixation indices near to zero (Table 3). As in the diploids, the tetraploid populations showed no evidence of realized inbreeding, in terms of inflated homozygosity.

\section{Genetic divergence from diploid populations}

Figure 2 is a dendrogram based on UPGMA cluster analysis of Nei's genetic distance among the tetraploid and diploid populations. The overall shape of the dendrogram with Jerrabomberra and Manor as the two most distinctive populations, both diploid, remains unchanged from that for the diploids alone (Young et al., 1999). The polyploid populations are nested within the diploid array. This would be expected if they are not genetic isolates and represent repeated samples from diploid progenitors.

\section{Discussion}

Indications that certain populations of $R$. leptorrhynchoides were tetraploid first came from preliminary zymograms in which balanced and unbalanced heterozygotes for various systems were clearly evident, and where some individuals appeared to contain more than two allozyme alleles at a locus. In addition, the same phenomenon was seen for several different isozyme systems in the same individuals or populations and not in others. Later, cytological examination of a large number of individuals (Young \& Murray, unpub. data) confirmed that the species was cytologically complex. Furthermore, meiotic configurations suggested that these populations were autoploid. It is, however, important to test the nature of the segregation at both the cytological and genic levels (Shore, 1991). We therefore sought evidence from segregation in controlled crosses.

\section{Segregation in tetraploid Rutidosis}

The gene marker data confirm that tetraploid $R$. leptorrhynchoides plants are autopolyploid. This is

(C) The Genetical Society of Great Britain, Heredity, 85, 122-129. 
Fig. 1 Relationship between size (on a log scale) of diploid and tetraploid populations of Rutidosis leptorrhynchoides and allelic richness, where $\Delta$ are northern diploid populations (from NSW and ACT), $\boldsymbol{\Delta}$ are Victorian diploid populations, and $\boldsymbol{C}$ are tetraploid populations (Victorian — see Table 1). The arrows indicate the displacement to account for double gene copy number in tetraploids for comparison with diploids. The regression line is for all diploid populations only $\left(r^{2}=0.56\right.$, $P<0.001)$.
Fig. 2 UPGMA cluster analysis of allozyme allele frequencies in Rutidosis leptorrhynchoides populations in south-eastern Australia. The populations from the southern set (in Victoria) are shaded and among those, the polyploid populations have oval outlines, whereas all diploids are rectangular boxes. The thick bar indicates $1 \mathrm{SE}$ of genetic distance for that taxon or cluster.
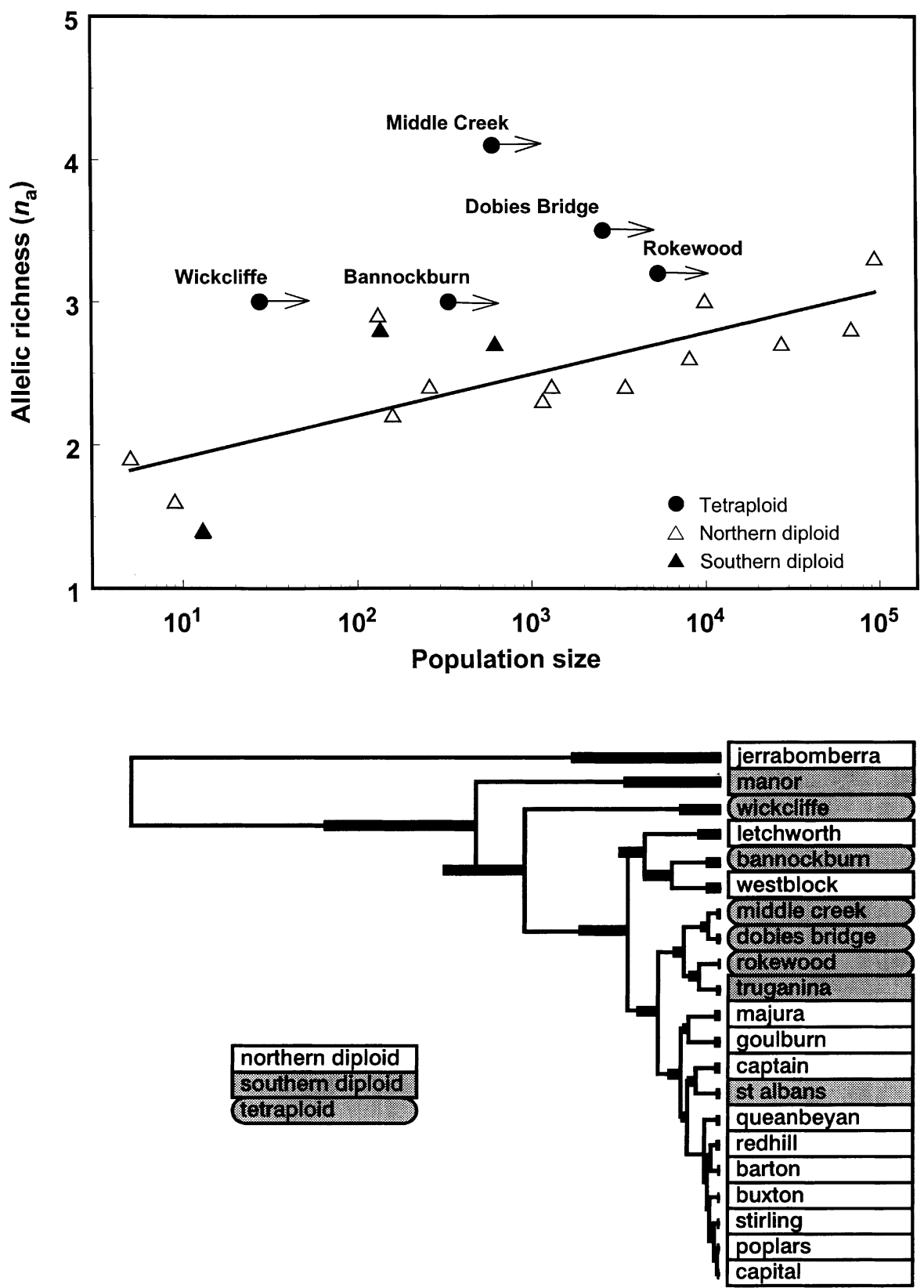

\section{Nei's genetic distance}

an important point to establish in assessing genetic variability. Individuals possess four copies of the one haploid genome, and the ability to form quadrivalents forces all the alleles of a locus into a single segregational unit. This increases the flexibility of the genetic system to generate a wider array of genotypes than is the case with strict disomic inheritance. Indeed 'fixed' heterozygosity is a state not open to a tetrasomic system. On the other hand, the tetrasomic system is conservative in the rapidity with which allele frequencies can change, or fixation is reached (Bever \& Felber, 1992).

\section{Measuring variability in tetraploid populations and its expectation}

Tetrasomy presents the geneticist with a numerical problem of how to score a quantity like allelic richness and compare it across ploidy types and levels. Consider

(C) The Genetical Society of Great Britain, Heredity, 85, 122-129. 
for example a hypothetical locus $Y d h 1$ in an allotetraploid species with disomic inheritance. For the locus $Y d h 1$, the species has two homoeologous loci, Ydh1A and $Y d h 1 B$, inherited from the two diploid parental species. Suppose two allozyme monomers only are observed in the population (e.g. $F$ fast and $S$ slow). In addition, the sample contains three distinct genotypes: FFSS, FSSS and SSSS. This suggests that both allozymes occur at one locus (e.g. $Y d h 1 A$ ) whereas the other locus is monomorphic. It would be logical to conclude that the allelic richness in this case was 1.5 alleles per locus. On the other hand, the same population data at this locus in an autotetraploid species, namely two allozymes, leads to the estimate of allelic richness as two alleles for this locus. It appears that tetrasomy per se has increased the estimate of allelic richness.

However, in such a computation, autotetraploids have double the number of gene copies per locus in both the population and the sample that diploids and allotetraploids have. Use of the sampling theory for the neutral allele model of Kimura \& Crow (1964) is one way to take account of both population and sample size in the comparison of tetraploid with diploid populations. In that model, the equilibrium distribution of allele frequency is a function of the single combined parameter $\theta=4 N u$ where $N$ is the population size and $u$ is the mutation rate. In a sample of $S$ random gametes, the expected number of selectively neutral alleles $(k)$ at a locus is approximately:

$k \approx \theta \log _{\mathrm{e}}[(S+\theta) / \theta]+0.6$

where $4 N u>0.1$ and $S>10$ (Brown, 1989). If the sample size in terms of the number of plants is similar in the diploid and autotetraploid data for both $N$ and $S$, then the expected allelic richness per locus in the autotetraploid species is about double that of the diploid populations. In the example, a $k$-value for the allotetraploid of 1.5 is equivalent to an allelic richness of $2 \times(1.5)-0.6=2.4$ in an autotetraploid.

The estimated allelic richness per locus in the tetraploid Rutidosis populations exceeded that in the diploid Victorian populations by only $30 \%$. If the expectation of richness was double that in diploids for the same size, we would infer that the tetraploids were depauperate in variation compared with diploid populations from the same region.

However, actual allelic richness in natural populations as observed for allozymes in plant species is more often positively related to the logarithm of population size (Young et al., 1996) than to absolute size as predicted in the neutral theory. This also holds for the diploid populations of $R$. leptorrhynchoides. This means that each tetraploid point should be displaced in Fig. 1 by a set increased distance along the $x$-axis for comparison with diploids for doubling the size. When this is done, as the arrows in Fig. 1 show, all five tetraploid populations still fall well above the regression line estimated for all diploid populations. Thus correcting for the general trend of allelic richness to relate to population size on a log scale leads to the conclusion that allelic richness in these tetraploid populations is at least comparable with, if not higher than, that in their diploid counterparts.

Does this increased variation indicate that tetraploid populations are double in genetic effective size? Sun \& Sun (1996) have suggested that fewer populations of tetraploid Spiranthes than of diploids are needed to achieve the same conservation goals. However, differences in other life-cycle attributes beyond the increased allelic richness would have to be assessed before minimum viable population size should be reduced for these autotetraploid populations.

\section{Divergence between tetraploids and diploids}

Cluster analysis of the isozyme variation in the two kinds of populations (Fig. 2) supports the notion that the tetraploids are not differentiated from the diploids in allelic content. Instead the result argues that gene flow occurs between the two ploidy levels. The cytological finding of aneuploid plants (Young \& Murray, 2000) further strengthens this hypothesis. In such a case, there are no grounds for actively stopping natural gene flow. Indeed autotetraploids presumably continue to arise spontaneously from diploid individuals, a process that would elevate gene flow between ploidy levels.

\section{Conclusions}

The conservation of the southern populations of $R$. leptorrhynchoides presents a number of stark choices that arise from the fact that a significant fraction of diversity in this species is contained in autotetraploid populations. The fact that in Victoria the tetraploids outnumber diploids, argues that they merit conservation in their own right and should not be overlooked. On the other hand, the genetic similarity with diploids and the occurrence of aneuploids indicates a long association in which diploids are probably a source of extra allelic diversity. Active extirpation of diploid populations from Victoria is not therefore supported. Retaining any extant zones of overlap will aid the generation of new tetraploid lineages and gene flow among them, and permit occasional gene flow between diploids and tetraploids for future flexibility. 
However, procedures for replenishing populations or founding new ones are another matter. The high sterility of triploid plants argues that strategies for replenishing and founding should avoid creating mixed ploidy situations, as they are likely to be counterproductive and wasteful of expensive resources.

While reviewing the genetic aspects of founding populations, Clegg \& Brown (1983) stressed that ploidy level must be regarded as an important variable in founding plant populations. They considered that deliberately increasing the level of ploidy with agents such as colchicine to conserve a species would be inappropriate. However, in species with mixed ploidy, artificially generated tetraploids might be the only source of seed to replace an extreme loss of autotetraploid populations. If such a strategy is implemented in $R$. leptorrhynchoides, more than one lineage may be needed to ensure an adequate diversity of self-incompatibility alleles.

\section{Acknowledgements}

We thank Drs Rex Oram and Walter Kelman for commenting on a previous draft, John Morgan for providing the site locations and help with seed collection, Liz Gregory for assistance with allozyme analyses and Department of Natural Resources \& Environment, Victoria for permission to collect seed.

\section{References}

Allard, R. W. 1960. Principles of Plant Breeding. John Wiley \& Sons Inc., New York.

ARFT, A. M. AND RANKER, T. A. 1998. Allopolyploid origin and population genetics of the rare orchid Spiranthes diluvialis. Am. J. Bot., 85, 110-122.

BAYER, R. J. 1989. Patterns of isozyme variation in western North American Antennaria (Asteraceae: Inulae). 1. Diploid and tetraploid species of section Alpinae. Am. J. Bot., 76, 679-691.

BEVER, J. D. AND FELBER, F. 1992. The theoretical population genetics of autopolyploidy. In: Antonovics, J. and Futuyma, D. (eds) Oxford Surveys in Evolutionary Biology, pp. 185-217. Oxford University Press, Oxford.

BRIGGS, J. D. AND LEIGH, J. H. 1995. Rare or Threatened Australian Plants. CSIRO Publishing, Melbourne.

BROWN, A. H. D. 1989. Core collections: a practical approach to genetic resources management. Genome, 31, 818-824.

CLEGG, M. T. AND BROWN, A. H. D. 1983. The founding of plant populations. In: Schonewald-Cox, C. M., Chambers, S. M., MacBryde, B. and Thomas, L. (eds) Genetics and Conservation, pp. 216-228. Benjamin/Cummings, Menlo Park, CA.

CONTE, L., TROIA, A. AND CRISTOFOLINI, G. 1998. Genetic diversity in Cytisus aeolicus Guss. (Leguminosae), a rare endemite of Italian flora. Plant Biosystems, 132, 239-249.
CROPPER, S. C. 1993. Management of Endangered Plants. CSIRO Publishing, Melbourne.

GLOVER, B. J. AND ABBOTT, R. J. 1995. Low genetic diversity in the Scottish endemic Primula scotica Hook. New Phytol., 129, 147-153.

HOKANSON, K. AND HANCOCK, J. 1998. Levels of allozymic diversity in diploid and tetraploid Vaccinium sect. Cyanococcus (blueberries). Can. J. Plant Sci., 78, 327-332.

KIMURA, M. AND CROW, J. F. 1964. The number of alleles that can be maintained in a finite population. Genetics, 49, 725-738.

LEITCH, I. J. AND BENNETT, M. D. 1997. Polyploidy in angiosperms. Trends Plant Sci., 2, 470-476.

PROBER, S. M., SPINDLER, L. H. AND BROWN, A. H. D. 1998. Conservation of the grassy white box woodlands: Effects of remnant population size on genetic diversity in the allotetraploid herb Microseris lanceolata. Conserv. Biol., 12, 1279-1290.

RAMSEY, J. AND SCHEMSKe, D. W. 1998. Pathways, mechanisms, and rates of polyploid formation in flowering plants. Ann. Rev. Ecol. Syst., 29, 467-501.

RANKer, T. A. AND ARFT, A. M. 1994. Allopolyploid species and the U.S. endangered species act. Conserv. Biol., 8, 895-897.

RITLAND, K. 1989. Genetic differentiation, diversity and inbreeding in the mountain monkeyflower (Mimulus caespitosus) of the Washington Cascades. Can. J. Bot., 67, 2017-2024.

SHORE, J. S. 1991. Tetrasomic inheritance and isozyme variation in Turnera ulmifolia vars. elegans Urb. \& intermedia Urb. (Turneraceae). Heredity, 66, 305-312.

SOltis, D. E. AND SOLTIS, P. M. 1999. Polyploidy: Recurrent formation and genome evolution. Trends Ecol. Evol., 14, 348-352.

SUN, M. AND SUN, M. 1996. Effects of population size, mating system and evolutionary origin on genetic diversity in Spiranthes sinensis and S. hongkongensis. Conserv. Biol., 10, 785-795.

THRALL, P. H. AND YOUNG, A. G. 2000. AUtOTET: a program for analysis of autotetraploid genotypic data. J. Hered., in press.

YOUNG, A., BOYLE, T. AND BROWN, T. 1996. The population genetic consequences of habitat fragmentation for plants. Trends Ecol. Evol., 11, 413-418.

YOUNG, A. G. AND BROWN, A. H. D. 1999. Increased paternal correlation in fragmented populations of the grassland daisy Rutidosis leptorrhynchoides. Genet. Res., 73, 111-117.

YOUNG, A. G. , BROWN, A. H. D. AND ZICH, F. A. 1999. Genetic structure of fragmented populations of the endangered daisy Rutidosis leptorrhynchoides. Conserv. Biol., 13, 256-265.

YOUNG, A., Miller, C., GREGORY, E. AND LANGSTON, A. 2000. Sporophytic self-incompatibility in diploid and tetraploid races of Rutidosis leptorrhynchoides (Asteraceae). Aust. J. Bot., in press.

YOUNG, A. G. AND MURRAY, B. G. 2000. Genetic bottlenecks and dysgenic gene flow into re-established populations of the grassland daisy Rutidosis leptorrhynchoides. Aust. J. Bot., 48, 409-416. 